\title{
Clarification and confocal imaging of the nonhuman primate placental micro-anatomy
}

$\overline{\text { JA Sargent*,1,2 , VHJ Roberts }{ }^{2}, \text { JE Gaffney² \& AE Frias }}{ }^{1,2}$

\section{ABSTRACT}

Geometry of the placental villous vasculature is a key determinant of maternalfetal nutrient exchange for optimal fetal growth. Recent advances in tissue clarification techniques allow for deep high-resolution imaging with confocal microscopy; however, the methodology lacks a signal:noise ratio of sufficient magnitude to allow for quantitative analysis. Thus, we sought to develop a reproducible method to investigate the $3 \mathrm{D}$ vasculature of the nonhuman primate placenta for subsequent data analysis. Fresh placental tissue was dissected, formalin fixed, clarified using a modified Visikol ${ }^{\circledR}$ protocol and immunolabeled for CD31 (fetal endothelium) and cytokeratin-7 (villous trophoblast) for confocal imaging of the microanatomy. We present a detailed clarification and staining protocol augmented for imaging of nonhuman primate placental tissue. The image stacks generated by this refined staining method and our data acquisition parameters can be analyzed quantitatively to provide insights regarding the villous and vascular microanatomy of the placenta.

\section{METHOD SUMMARY}

We present a protocol for the preparation, immunolabeling and clarification of the nonhuman primate placenta refined for confocal microscopy and subsequent quantification of the microanatomic structures.

KEYWORDS

confocal microscopy - immunohistochemistry $\cdot$ nonhuman primate • placenta $\cdot$ tissue clarification

'Department of Obstetrics \& Gynecology, Oregon Health \& Science University, Portland, OR, USA; ${ }^{2}$ Division of Reproductive \& Developmental Sciences, Oregon National Primate Research Center, Beaverton, OR, USA; *Author for correspondence: sargenja@ohsu.edu

BioTechniques 66: 79-84 (February 2019) 10.2144/btn-2018-0110
To ensure adequate gas exchange and transportation of nutrients to the fetus, the appropriate development of the maternal and fetal placental vascular networks is essential $[1,2]$. The caliber, branching patterns, and interrelationships of the fetal vasculature and the terminal and stem villi are thought to be essential for maintaining adequate uniform blood supply throughout the placenta. Dysfunctional alterations in these morphologies or relationships have been linked to post-placental hypoxia and subsequent intrauterine growth restriction, hypertensive disorders of pregnancy and stillbirth [1-8].

Until recently, assessment of the placental vasculature has largely relied upon stereological or vascular casting methods. Traditional stereology utilizes paraffin-embedded tissue slices and reproducible counting methods to estimate variables such as villous or vessel size, number, surface area and volume; more advanced computational methods can then be employed to estimate diffusion distance and complex hemodynamic parameters [8-11]. While stereology allows the observer to assess for additional histologic variables (e.g., syncytial knots, placental infarcts and villous maldevelopment), it is limited to a 2D view without preserving the $3 \mathrm{D}$ vascular and villous geometry $[1,2,12]$. To address these issues, corrosion casting of the placental vasculature was developed as a means to preserve the vascular morphology for qualitative and quantitative assessment [13-17]. Unfortunately, these methods are technically complex, they can lead to distortion or destruction of the fetal capillaries, and because of the corrosive tissue digestion process, they eliminate the potential to evaluate the relationship between the fetal vascular bed and the surrounding villous tissue [14]. As such, new methods are required that allow for the investigation of the 3D vascular structure and the villous architecture.
Through the imaging of immunofluorescent-labeled and clarified tissue with a confocal microscope, new approaches are emerging that address the shortfalls of stereological and vascular casting methods. The use of confocal microscopy to capture precisely located optical sections allows for the $3 D$ rendering of the images [18-20]; however, the depth of tissue analysis has been limited due to refractive index mismatching, subsequent light-scattering, and auto-fluorescence [21]. The clarification of biologic specimens through refractory index equilibration has been studied since the beginning of the 20th Century, however earlier methods were damaging to tissue and incompatible with fluorescently labeled specimens [22]. To take advantage of the increasingly sophisticated confocal methodologies available, newer tissue clarification methods have been developed that are either based on hydrogel embedding, hyper-hydration, simple immersion or solvent-based techniques; each of these having their respective deleterious effects on tissue structure, or compatibility issues with immunofluorescently labeled tissue [22]. Visikol ${ }^{\circledR}$ HISTO $^{\text {TM }}$ is a proprietary reversible clearing technique that uses an ethanolbased solvent, which causes minimal tissue alteration (shrinkage/expansion of $<5 \%$ ) and is compatible with immunofluorescent staining. Recently, Visikol HISTO has been utilized to generate $3 \mathrm{D}$ renderings of immunofluorescent-labeled and clarified human placental tissue $[23,24]$, and this has generated interest in customizing its use for novel analyses. Unfortunately, protocols refined for human application did not yield stacks of sufficient quality so as to allow for subsequent reliable quantitative analysis when applied to the nonhuman primate placenta. Specifically, chemical tissue fixation with Zn Formalin is required to ensure microanatomic structure stabilization during the long incubation times required for deep tissue 


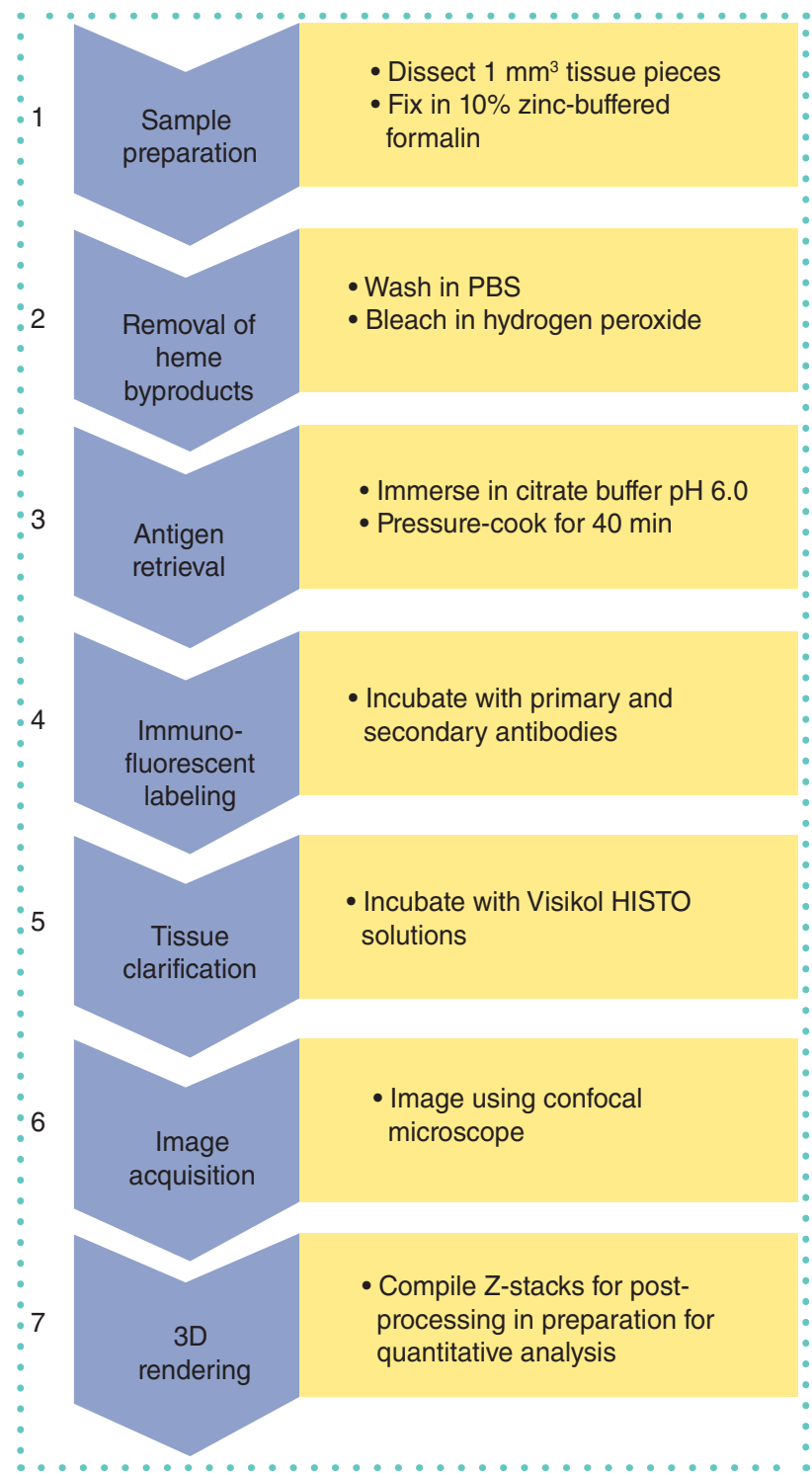

Figure 1. Workflow for protocol.

PBS: Phosphate-buffered saline.

- imaging. The subsequent molecular cross-linking masks tissue antigens that are of scientific interest, and antigenspecific antigen retrieval steps must be optimized in order to allow for successful immunofluorescent labeling. Additionally, when exposed to laser excitation, many biologic tissues contain molecules (e.g., elastin, collagen, lipofuscin and heme byproducts) that fluoresce within the visible wavelengths $(350-630 \mathrm{~nm})$; if not reduced, this auto-fluorescence creates optical noise and interferes with quantitative analyses. Photo-bleaching of natural fluorophores can be accomplished in a wavelength-specific manner through photo-induced free-radical generation and chemical destruction using light- emitting diodes (LEDs) without disrupting the tissue integrity [25]. This has not been achieved in placental confocal imaging studies to date.

Numerous computer programs have been created to process confocal images and allow for image optimization, 3D rendering and subsequent analysis. ImageJ is a versatile, Java-based, image-processing, free software developed by the $\mathrm{NIH}$, which has a flexible development model allowing for simple user-generated plug-ins to increase the programs functionality $[18,26]$. The 3D imagebased quantification of vascular networks has been approached in various fields of medicine [27], and Image J has a multitude of plugins that have been validated for this use making it suitable for use in our study [28].

Placental and fetal development is similar between humans and nonhuman primates (NHPs), and NHPs have been used successfully as a translational model to human pregnancies. Numerous validated, wellcharacterized, highly controlled NHP pregnancy disease models exist providing a unique opportunity to assess underlying disease mechanisms and potential therapeutic strategies. Using confocal imaging methods, quantified analysis of the NHP placenta has not yet been accomplished; however, this could provide critical insights into both human and NHP pregnancies. Our objective was to develop and refine a protocol for the immunofluorescent labeling and clarification of the placenta in the NHP to allow for high-resolution 3D imaging of sufficient quality for advanced quantification methods (Figure 1).

\section{MATERIALS \& METHODS}

Figure 1 provides a schematic overview of the seven stages of our protocol as outlined in detail below.

\section{Sample preparation}

All animal procedures were conducted in accordance with the guidelines of the Institutional Animal Care and Use Committee (IACUC) of the Oregon National Primate Research Center (ONPRC). The ONPRC abides by the Animal Welfare Act and Regulations enforced by the United States Department of Agriculture. Adult female rhesus macaques underwent time-mated breeding to generate pregnancies from which placental tissue was obtained. All animals were pair housed and maintained on a control standard chow diet with additional access to daily enrichment foods and foraging devices. All placental tissues were obtained under general anesthesia at the time of cesarean section delivery at gestational day 140 (term being 168 days), immediately placed in phosphate-buffered saline (PBS), and the decidua and membranes dissected away from the villous tissue. $1 \mathrm{~mm}^{3}$ tissue sections were isolated and immersed in $10 \%$ zinc formalin for $4 \mathrm{~h}$ prior to transfer in $70 \%$ ethanol and storage at $4^{\circ} \mathrm{C}$. For the development of this protocol we utilized over 300 samples from 20 NHP pregnancies. Shorter formalin exposure times were associated with incomplete fixation, while longer incubations resulted in proportional worsening of the background auto-fluorescence.

\section{Removal of heme byproducts}

To address background fluorescence caused by hemoglobin byproducts, fixed tissue was placed in PBS and gently rocked at $4^{\circ} \mathrm{C}$ (Boekel Rocker II 260350-2 Platform Rocker, Boekel Scientific, PA, USA) for $48 \mathrm{~h}$. Tissue was dehydrated with 50,75 and $100 \%$ methanol washes with gentle rocking at $4^{\circ} \mathrm{C}$ for 10 min per wash. Using a bleaching solution $\left(30 \% \mathrm{H}_{2} \mathrm{O}_{2}\right.$, dimethyl sulfoxide [DMSO], methanol in a 1:1:4 ratio), tissue was incubated at $4^{\circ} \mathrm{C}$ for $48 \mathrm{~h}$ under gentle rocking. We found that the $48-\mathrm{h}$ exposure times, with agitation, were required to remove any retained hemoglobin byproducts, which otherwise create excessive background noise and 

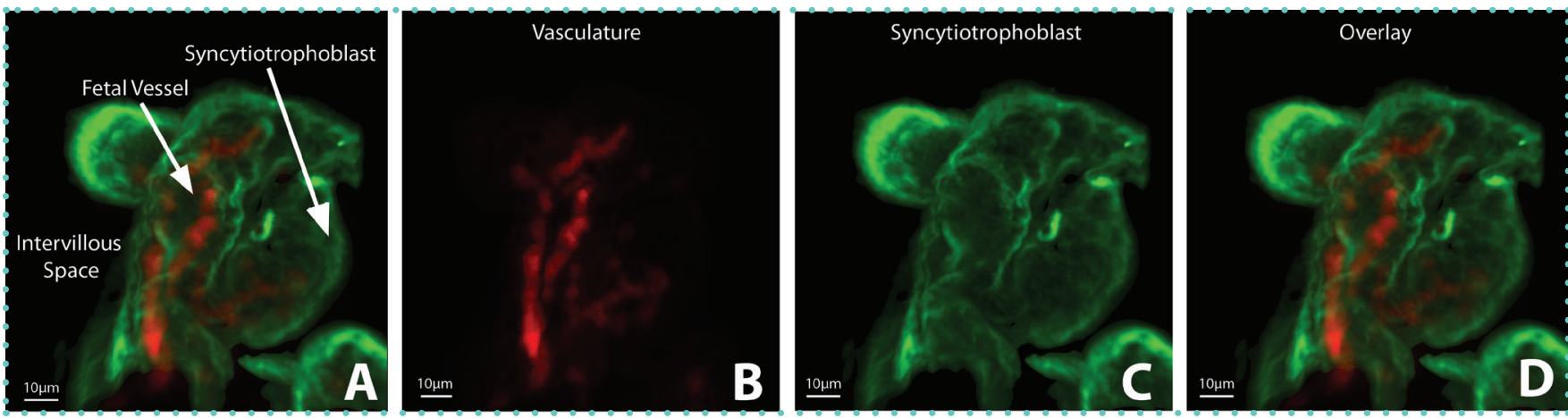

Figure 2. Maximum-intensity projections of immunofluorescent-labeled (CD31 - red; CK7 - green) and clarified NHP placental tissue imaged using the Leica SP5 AOB5 spectral confocal system with a 20x PL APO NA 0.75 AIR CORR CS2 objective. (A) Placental tissue visualized at excitation wavelengths for the detection of (B) CD31 (AF647), (C) CK7 (AF488), and (D) overlay.

NHP: Nonhuman primate.

unacceptably interfere with quantitative analysis. To reduce general auto-fluorescence, heat exposure was limited and all steps were performed under LED bulbs with wavelengths of $480 \mathrm{~nm}$ and $660 \mathrm{~nm}$ (45W Multi-Spectrum LED, iPower, CA, USA) at a distance of $6 \mathrm{~cm}$.

\section{Antigen retrieval}

For immunofluorescent staining, antigen retrieval was essential. Tissue was rehydrated using 20\% DMSO/methanol, 75 and $50 \%$ methanol, and PBS washes with gentle rocking at $4^{\circ} \mathrm{C}$ for $10 \mathrm{~min}$ per wash. Optimal antigen retrieval was obtained using Citrate Buffer (10 mM citric acid with $0.05 \%$ Tween at $\mathrm{pH}$ 6) exposed to $80^{\circ} \mathrm{C}$ at $6 \mathrm{psi}$ (Cuisenart Electric Pressure Cooker CPC600, Cuisenart, NJ, USA) for $40 \mathrm{~min}$, and then placed at $21^{\circ} \mathrm{C}$ for $20 \mathrm{~min}$. Antigen retrieval times of 10,20, 40 and 60 min were tested and heat and pressure conditions were varied. While the $\mathrm{CK} 7$ immunofluorescent labeling was robust over the breadth of trials, the final exposure conditions were required to optimize the $C D 31$ labeling while minimizing the worsening background autofluorescence caused by the heat and pressure conditions. Given the specific optimization required for our two antigens, it is likely that a variety of conditions will need to be tested in order to modify this protocol if alternative structural labels are explored by this method.

\section{Immunofluorescent labeling}

Blocking and permeabilization was performed using a solution of $2 \%$ Donkey Serum and $0.2 \%$ Triton $\mathrm{X}-100$ in PBS with gentle rocking at $4^{\circ} \mathrm{C}$ for $24 \mathrm{~h}$ under LED exposure at $4^{\circ} \mathrm{C}$. A primary antibody

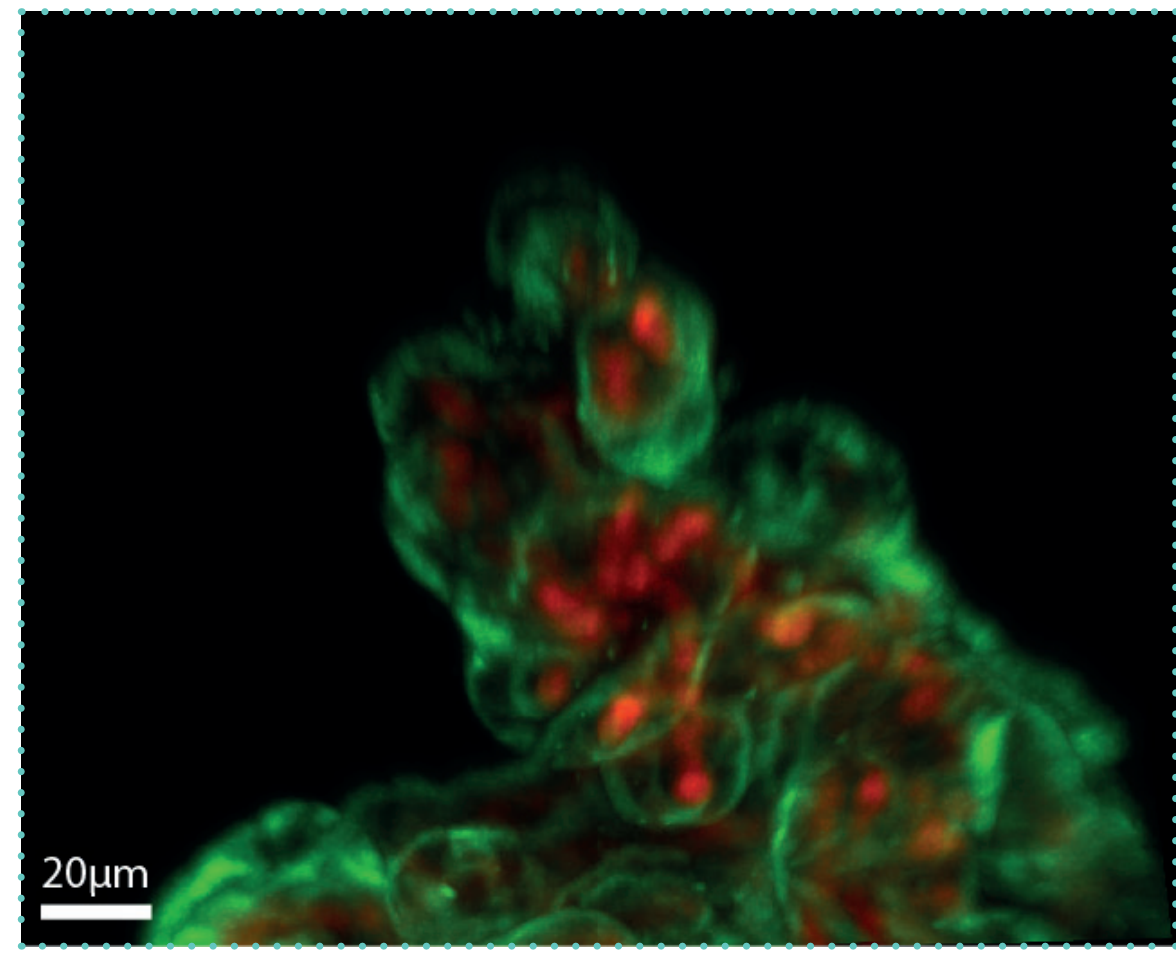

Figure 3. Maximum-intensity projection of immunofluorescent-labeled (CD31 - red; CK7 - green) and clarified NHP placental tissue imaged using the Leica SP5 AOB5 spectral confocal system with a 20x PL APO NA 0.75 AIR CORR CS2 objective. NHP: Nonhuman primate.

solution containing both a monoclonal mouse-anti-human CD31 (Thermofisher MA1-26196, endothelial cell marker) and a polyclonal rabbit-anti-human CK7 (Abcam, ab103687, trophoblast marker) was made at 1:10 concentrations in a solution of $2 \%$ donkey serum in PBS, and tissue was immersed and placed at $37^{\circ} \mathrm{C}$ for $72 \mathrm{~h}$. Four 10 min washes of $2 \%$ Donkey Serum in PBS at $21^{\circ} \mathrm{C}$ were then performed before placing in a secondary antibody solution of donkeyanti-mouse pre-adsorbed polyclonal antibody (Abcam, ab150109, alexafluor 488) and a donkey-anti-rabbit antibody (Abcam, ab150075, alexafluor 647) at a concentration of 1:250 for both secondaries in $2 \%$ Donkey Serum and PBS for $12 \mathrm{~h}$ at $21^{\circ} \mathrm{C}$ with agitation at $150 \mathrm{rpm}$. Shorter incubation times and altered temperatures $\left(4,21\right.$ and $37^{\circ} \mathrm{C}$ were trialed) were associated with incomplete tissue penetration as assessed by subjective observation at the time of imaging where antibody-positive staining was visualized on the edge of placental tissue and not within the villous structure. 


\section{- Tissue clarification}

Samples were washed four times in $2 \%$ donkey serum and PBS for $10 \mathrm{~min}$ at $21^{\circ} \mathrm{C}$ at $150 \mathrm{rpm}$ prior to dehydration. Three 10 -min washes of 50,75 and $100 \%$ methanol were performed before immersing the tissue in the Visikol HISTO1 for $4 \mathrm{~h}$ at $21^{\circ} \mathrm{C}$ at $150 \mathrm{rpm}$. The tissue was placed in the Visikol HISTO2 solution overnight, prior to imaging the next day.

\section{Image acquisition}

Clarified samples were placed in a $12-\mathrm{mm}$ Nunc $^{\mathrm{TM}}$ Glass Bottom Dish (Thermofisher Scientific, TX, USA), and immersed in 500 $\mu$ of Visikol HISTO2 solution. Imaging was performed using a Leica SP5 AOB5 spectral confocal system in two-channel (Leica/ ALEXA 488, Leica/ALEXA 633) optical sections at optimized intervals calculated by the imaging system $(\sim 1 \mu \mathrm{m}$ per slice, 200-300 slices per sample) using a x20 PL APO NA 0.75 IMM CORR CS2 air objective. Optical detectors were set to $488-520 \mathrm{~nm}$ and $635-680 \mathrm{~nm}$, respectively, to minimize crossover. To increase resolution, the confocal was set to $1024 \times 1024$ pixels, pinhole of 0.4 Airy, at $200 \mathrm{~Hz}$ acquisition speed, with line averaging. Negative controls (e.g., no primary antibody, or no secondary antibody) were used to adjust the confocal gain sensitivity to eliminate auto-fluorescence.

\section{$3 \mathrm{D}$ rendering}

Imaged z-stacks were uploaded into FijiImage J as hyperstacks and the stacks were projected into either maximum intensity or $3 \mathrm{D}$ renderings.

\section{RESULTS \& DISCUSSION}

We describe the developed protocol for the successful immunofluorescent labeling and clarification of the placenta of the NHP, which has been refined for confocal microscopy and quantitative analysis. We approached protocol development as an iterative process with the aim to maximize both reproducibility and the signal-to-noise ratio of our immunofluorescent labeling as well as to minimize the need for post-imaging processing. Our predetermined threshold for resolution was $3 \mu \mathrm{m}$; the terminal diameter of the capillary vessels. When visualized at 20x magnification (PLAPO NA 0.75 AIR CORR CS2 objective), maximumintensity projections of the clarified and immunolabeled NHP placental tissue allow for clear delineation of the capillary vessels from the syncytiotrophoblast tissue (Figure 2), and the 3D orientation of the placental micro-anatomy within the

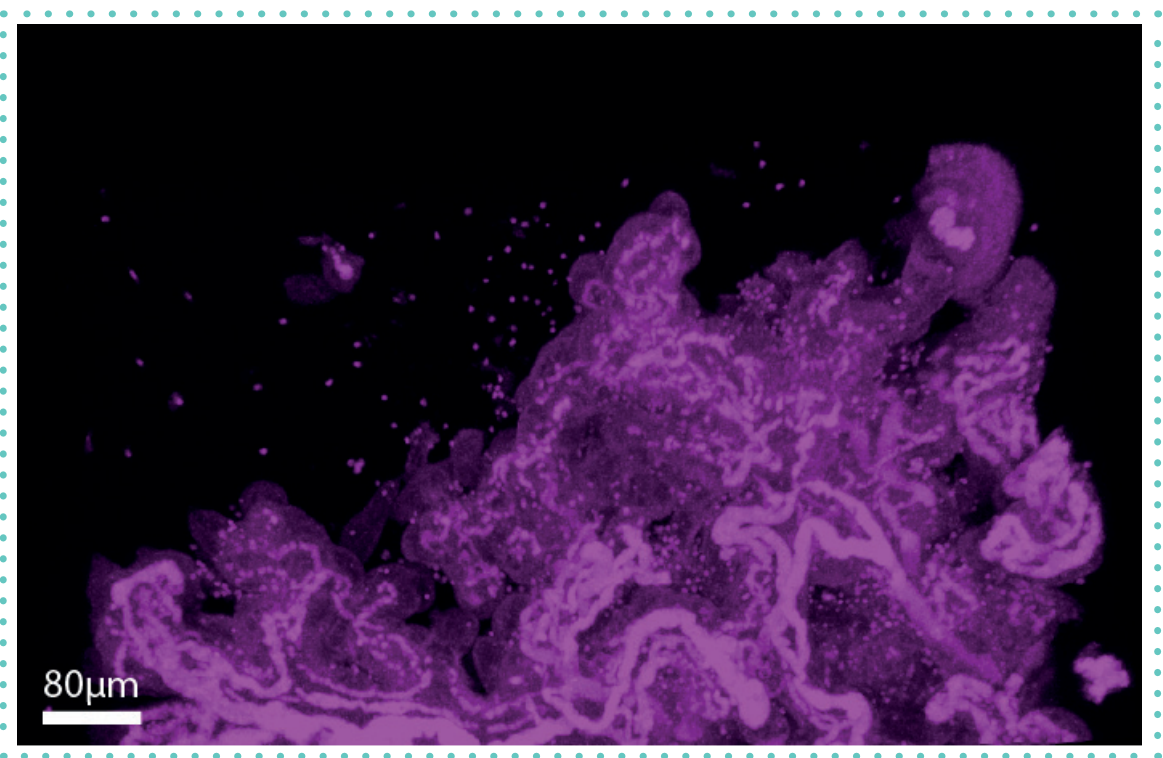

Figure 4. Demonstration of tissue auto-fluorescence and contamination by heme byproducts. Maximumintensity projection of fixed, unlabeled, nonclarified placental tissue of the NHP imaged using the Leica SP5 AOB5 spectral confocal system with a 20x PL APO NA 0.75 AIR CORR CS2 objective at 512 $\mathrm{nm}$. Arterioles are high-intensity due to elastin auto-fluorescence while hemoglobin byproducts are visualized as 'Christmas lights' throughout the image. Villous tissue is visible secondary to natural auto-fluorescence from lipofuscin and other organic molecules. NHP: Nonhuman primate. intervillous space (Figure 3). While other groups have used the Visikol HISTO solvent for placental clarification, our group found that those protocols were ineffective in the NHP placenta. Specifically, we found that antigen retrieval was essential, and multiple measures were required to decrease autofluorescence and maximize the intensity of the immunofluorescent labeling.

\section{Experimental considerations}

Auto-fluorescence

Fixed placental tissue, visualized with confocal microscopy in the absence of clarification or immunolabeling, has an abundance of natural auto-fluorescent noise (generated through laser-excitation of fluorophores in lipofuscin, elastin, collagen, heme byproducts and byproducts of cellular metabolism) making it unsuitable for quantitative analysis (Figure 4). Interfering autofluorescence can come from multiple sources including naturally occurring organic molecules and retained heme byproducts, and they can also be induced by the fixation process. While imaging techniques and post-imaging correction methods exist, these are unreliable and can reduce the desired signal from immunolabeled structures. For this protocol, duration of $\mathrm{Zn}$ Formalin exposure was minimized to decrease the amount of fixation-induced auto-fluorescence. Clearance of red blood cells can be achieved through saline perfusion of fresh placenta; however, this is technically difficult in the NHP and not always feasible. Rinsing the tissue with PBS followed by $\mathrm{a}_{2} \mathrm{O}_{2}$-based bleaching solution under optimized conditions reliably removed speckling from retained heme byproducts. Lastly, minimizing heat exposure and exposing the tissue to LED photobleaching further decreased the background natural and fixation-induced auto-fluorescence.

\section{Antigen retrieval}

We found antigen retrieval to be necessary for our CD31 and CK7 antigens, with the temperature, duration and $\mathrm{pH}$ optimized as a balance between the two antibodies. In addition, numerous alternative primary antibodies and lectins were trialed under varying conditions (concentration, agitation, duration, temperature) and antigen retrieval steps (buffer type, $\mathrm{pH}$, duration, temperature and pressure settings). Prolonged, high- 
concentration washes under heat were required to obtain optimal signal strength and tissue penetration.

\section{Clarification \& reversal}

Tissue clarification was carried out as per the manufacturer recommendations. While the use of the second solution (Visikol HISTO2) was listed as being optional for vascular tissues like the placenta, omitting this step dramatically decreased the effectiveness of the clarification process. Reversal of the clarification process and subsequent H\&E-stained paraffin-embedded slides were generated to correlate our quantitative analysis with stereologic findings.

\section{Image acquisition \& analysis}

Image $J$ is a freeware program capable of rendering uploaded z-stacks into $3 \mathrm{D}$ and subsequently analyzing the geometry of the placental micro-anatomy. While filters exist for the smoothing of immunolabeled structures and subtracting out background noise, we sought to minimize the amount of post-imaging processing required prior to analysis by maximizing the signal-tonoise ratio of our immunofluorescentlabeling and clarification protocol. Prior to image analysis, the vascular channel must be isolated and made binary, a process whereby each voxel is analyzed and those that have an intensity below a specified threshold are removed while those that are above threshold are altered to a uniform intensity (Figure 5). This crucial step requires accurate determination of the set threshold, and z-stacks containing heme byproducts, unevenly stained structures, or increased auto-fluorescence result in the loss of labeled structures and the incorporation of unwanted noise interpreted as structures of interest. Through the refinement of our protocol, ImageJ becomes more sensitive and specific to our immunolabeled objects of interest and the subsequent quantitative analysis is more robust.

\section{CONCLUSIONS \& FUTURE APPLICATION}

With the development of new analytic tools our ability to determine disease etiology and monitor response to therapy improves. The protocol as described in this paper allows for the generation of confocal imaged z-stacks with sufficient resolution to pursue
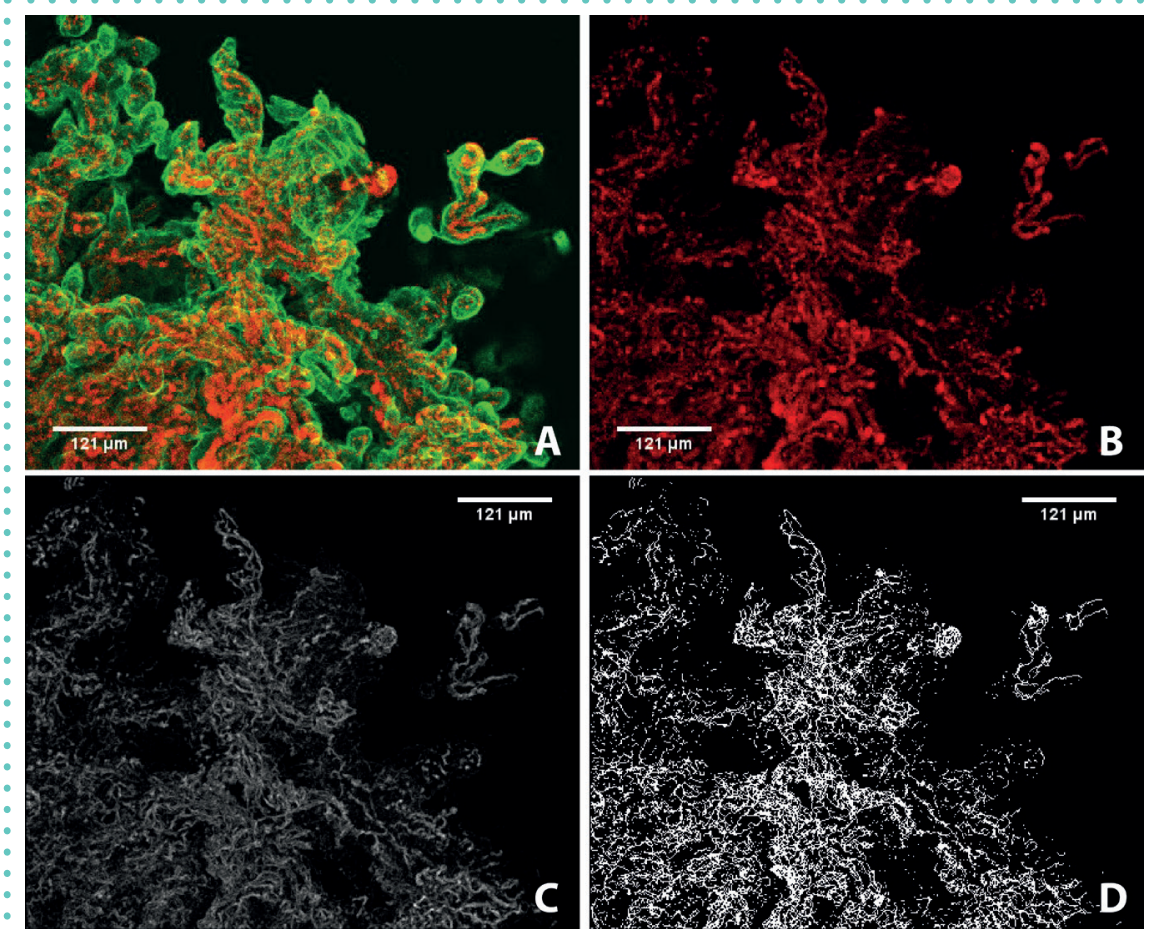

Figure 5. Maximum-intensity projections of immunofluorescent labeled (CD31 - red; CK7 - green) and clarified NHP placental tissue imaged using the Leica SP5 AOB5 spectral confocal system with a 20x PL APO NA 0.75 AIR CORR CS2 objective. (A) Placenta tissue visualized at excitation wavelengths for the detection of CD31 (AF647) and CK7 (AF488); (B) the same tissue following isolation of the fetal vasculature, and background subtraction (rolling ball radius of 10); (C) the same tissue once it has been made binary and undergone thresholding; (D) the same tissue following skeletonization, the final step prior to quantitative analysis.

reliable and reproducible quantitative analyses. By identifying and reliably quantifying micro-anatomic changes in highlycontrolled, pathologic NHP pregnancies, precise mechanisms can be identified, explored and monitored in the absence of the inevitable confounding variables that complicate analysis of human pregnancies. When these methods are subsequently applied to human placenta studies, insights from NHP pregnancies can potentially translate to clinical breakthroughs and improvement in obstetric outcomes.

\section{FUTURE PERSPECTIVE}

As progress in the confocal imaging field continues to advance, the availability of commercially available products (such as Visikol HISTO) will allow for improved consistency in protocol reproducibility across multiple scientific disciplines and research institutes. For placental research, we anticipate that modification of the protocol described here could potentially be used for other 3D analyses including the localization of viral and bacterial antigens, cells of the immune system, membrane proteins and transporters, or genetic alterations (e.g., placental mosaicism)

\section{AUTHOR CONTRIBUTIONS}

JAS, VHJR and AEF conceived the study and experimental design. JAS performed the experiments with the assistance of JEG. JAS performed data acquisition. JAS wrote the manuscript with assistance from VHJR. All authors reviewed and approved the final manuscript.

\section{FINANCIAL \& COMPETING INTERESTS DISCLOSURE}

Research reported in this publication was supported by the Eunice Kennedy Shriver National Institute of Child Health \& Human Development (NICHD) under Award Number R01-HD086331 (to AEF). The authors have no other relevant affiliations or financial involvement with any organization or entity with a financial interest in or financial conflict with the subject matter or materials discussed in the manuscript apart from those disclosed. 
- No writing assistance was utilized in the production of this manuscript.

\section{ACKNOWLEDGMENTS}

We would like to thank Eliot Spindel from the Oregon National Primate Research Center, George Merz from the New York State Institute for Basic Research in Developmental Disabilities, and Tom Villani from Visikol ${ }^{\circledR}$ for their support in developing this protocol.

\section{ETHICAL CONDUCT OF RESEARCH}

All animal procedures were conducted in accordance with the guidelines of the Institutional Animal Care and Use Committee (IACUC) of the Oregon National Primate Research Center (ONPRC). The ONPRC abides by the Animal Welfare Act and Regulations enforced by the United States Department of Agriculture.

\section{OPEN ACCESS}

This work is licensed under the Creative Commons Attribution-NonCommercialNoDerivatives 4.0 Unported License. To view a copy of this license, visit http://creativecommons.org/licenses/by-nc-nd/4.0/

\section{REFERENCES}

Papers of special note have been highlighted as: - of interest

1. Benirschke K. Pathology of the Human Placenta. Burton G, Baergen RN (Eds). Springer, Berlin, Germany, London, UK (2012)

2. Baergen RN. Manual of Pathology of the Human Placenta Second Edition. Springer US, Boston, MA, USA (2011).

3. Furuya M, Ishida J, Aoki I, Fukamizu A. Pathophysiology of placentation abnormalities in pregnancy-induced hypertension. Vasc. Health Risk Manage. 4(6), 1301-1313 (2008)
Excellent review that outlines the molecular and hormonal pathophysiologic mechanisms that underlie defects in placentation and the development of hypertensive disorders of human and mouse pregnancies.

4. Kovo M, Schreiber L, Elyashiv O, Ben-Haroush $A$ Abraham G, Bar J. Pregnancy outcome and placental findings in pregnancies complicated by fetal growth indicin 22(3), 316-321 (2015).

Retrospective review of 342 human pregnancies complicated by fetal growth restriction ( 72 with hypertensive disorders of pregnancy and $\mathbf{2 7 0}$ without). Investigators found high rates of placental vascular lesions (e.g., acute atherosis and mural hypertrophy) in both groups, and an increase in the number of villous lesions groups, and an increase in the number of villous lesions knocia villous aglutination, intervillous fibrin syncytial knots, villous agglutination, intervillous fibrin deposition and villous infarcts) in those restriction when compared with those pregnancies with restriction when compared
isolated growth restriction.

5. Ptacek I, Smith A, Garrod A et al. Quantitative assessment of placental morphology may identify specific causes of stillbirth. BMC Clin. Pathol. 16, 1 (2016).

- Comparison of 49 placentas from human pregnancies complicated by stillbirth with matched placentas from liveborn preterm and term pregnancies. In pregnancies complicated by stillbirth, investigators found decreased numbers of blood vessels, decreased proliferation and trophoblastic area, and increased numbers of avascular villi.

6. Rainey A, Mayhew TM. Volumes and numbers of intervillous pores and villous domains in placentas associated with intrauterine growth restriction and/or pre-eclamp sia. Placenta 31(7), 602-606 (2010).

7. Salafia CM, Yampolsky M, Misra DP et al. Placental surface shape, function, and effects of maternal and fetal vascular pathology. Placenta 31(11), 958-962 (2010).

8. Mayhew TM, Manwani R, Ohadike C, Wijesekara J, Baker PN. The placenta in pre-eclampsia and intrauterine growth restriction: studies on exchange surface areas,
diffusion distances and villous membrane diffusive conductances. Placenta 28(2-3), 233-238 (2007).

9. Peterson DA. Quantitative histology using confocal microscopy: implementation of unbiased stereology procedures. Methods 18(4), 493-507 (1999).

10. Mayhew TM, Ohadike C, Baker PN, Crocker IP, Mitchell $\mathrm{C}$, Ong SS. Stereological investigation of placental morphology in pregnancies complicated by pre-eclampsia with and without intrauterine growth restriction. Placenta 24(2-3), 219-226 (2003).

11. Plitman Mayo R, Charnock-Jones DS, Burton GJ, Oyen ML. Three-dimensional modeling of human placental terminal villi. Placenta 43, 54-60 (2016)

12. Haeussner E, Aschauer B, Burton GJ et al. Does 2D-histologic identification of villous types of human placentas at birth enable sensitive and reliable interpretation of 3D structure? Placenta 36(12), 1425-1432 (2015).

13. Ribatti D. Vascular Morphogenesis Methods and Protocols. Springer, NY, USA, Imprint: Humana Press, NY, USA (2015).
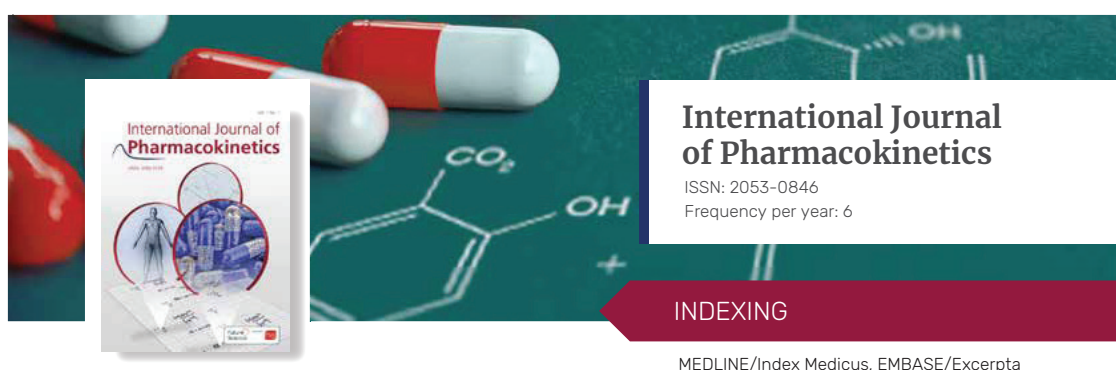

International Journal of Pharmacokinetics

ISSN: 2053-0846

Frequency per year: 6

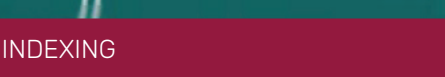

MEDLINE/Index Medicus, EMBASE/Excerpta Medica, Chemical Abstracts, Science Citation Index Expanded (SciSearch $^{\circledR}{ }^{\circledR}$ ). Biotechnology Citation Index ${ }^{\circledR}$, Current Contents ${ }^{\circledR} /$ Life Sciences, Journal Citation Reports/Science Edition $^{\circledR}$, Inspec ${ }^{\circledR}$, Scopus

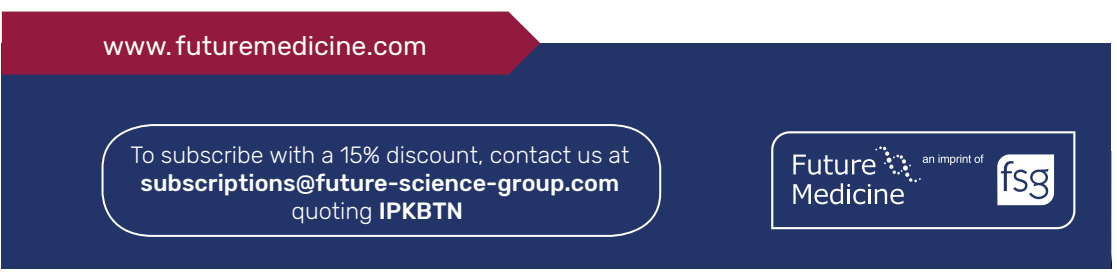

14. Whiteley KJ, Pfarrer CD, Adamson SL. Vascular corrosion casting of the uteroplacental and fetoplacental vasculature in mice. Methods Mol. Med. 121, 371-392 (2006).

15. Junaid TO, Bradley RS, Lewis RM, Aplin JD, Johnstone ED. Whole organ vascular casting and microCT examination of the human placental vascular tree reveals novel alterations associated with pregnancy disease. Sci. Rep. 7(1), 4144 (2017).

16. Yin $\mathrm{G}$, Chen $\mathrm{M}, \mathrm{Li} \mathrm{J}$ et al. Vascular corrosion casting of normal and pre-eclamptic placentas. Exp. Ther. Med. 14(6), 5535-5539 (2017)

17. Gong SP, Zhao YT, Yu YH. Vascular network modeling reveals significant differences in vascular morphology in growth-restricted placentas. Rev. Obstet. Gynecol. 4(3-4), 103-108 (2011)

Comparison of 20 placentas from human pregnancies complicated by fetal growth restriction and 20 control placentas using vascular casting methods. When compared with normal term pregnancies, the fetal growth restriction group was found to have decreased vascular branction group was found to have decreased vascular and smaller vessel diameters.

18. Sheppard C. Confocal Laser Scanning Microscopy. Shotton D (Ed.). Royal Microscopical, Oxford, UK; BIOS Scientific Publishers, NY, USA; Springer, Oxford, UK, NY, USA (1997).

19. Restini LMB. Current Microscopy Contributions to Advances in Science and Technology. Mendez-Vilas A (Ed.) Formatex Research Center, Badajoz, Spain (2012)

20. Price RL, Jerome WG. Basic Confocal Microscopy. Springer, NY, USA (2011)

21. Paddock SW. Confocal Microscopy Methods and Protocols. Springer, NY, USA, Imprint: Humana Press, NY, USA (2014).

22. Richardson DS, Lichtman JW. Clarifying tissue clearing. Cell 162(2), 246-257 (2015).

23. Merz G, Schwenk V, Shah R et al. Three-dimensional rendering and analysis of immunolabeled, clarified human placental villous vascular networks. J. Vis. Exp. (133), (2018).

- A video follow-up to the 2017 Placenta article by the same group that demonstrates the clarification and immunolabeling protocol in the human placenta and begins the discussion of the potential quantitative analyses possible with these techniques.

24. Merz G, Schwenk V, Shah RG, Necaise P, Salafia CM Clarification and $3-D$ visualization of immunolabeled human placenta villi. Placenta 53, 36-39 (2017).

- First reported use of Visikol ${ }^{\otimes}$ HISTO $^{\text {tw }}$ in the placenta. Investigators developed a protocol for the clarification and immunolabeling of the human placenta, and although these protocols do not work in the nonhuman primate - their findings marked an exciting step towards the quantitative analysis of the vascular and villous networks of the placenta.

25. Duong H, Han M. A multispectral LED array for the reduction of background autofluorescence in brain tissue. J. Neurosci. Methods 220(1), 46-54 (2013).

26. Hartig SM. Basic image analysis and manipulation in ImageJ. Curr. Protocols Mol. Biol. Chapter 14, Unit14.15 (2013).

27. Leahy C, Radhakrishnan $\mathrm{H}$, Weiner G, Goldberg JL, Srinivasan VJ. Mapping the 3D connectivity of the rat inner retinal vascular network using OCT angiography. inner retinal vascular network using OCT angiography.
Investig. Ophthalmol. Visual Sci. 56(10), 5785-5793 (2015).

28. Marks PC, Preda M, Henderson T et al. Interactive 3D analysis of blood vessel trees and collateral vessel volumes in magnetic resonance angiograms in the mouse ischemic hindlimb model. Open Med. Imag. J. 7 19-27 (2013). 